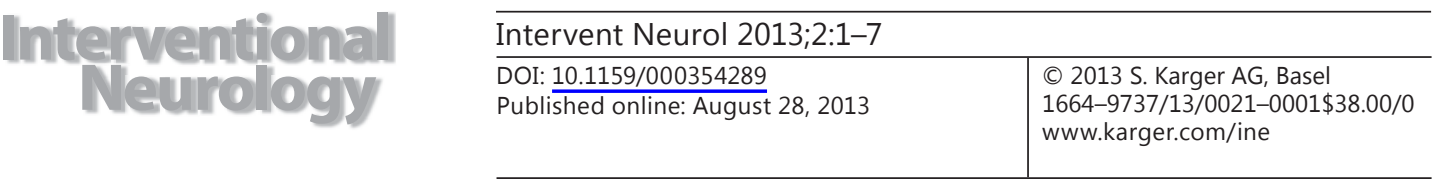

\title{
Dilation of the Internal Carotid Artery at the Entrance to the Carotid Canal following Carotid Artery Stenting Predicts Postprocedural Hyperperfusion
}

\author{
Norihito Shimamura ${ }^{a}$ Tomoshige Kikkawa $^{b}$ Mitsuaki Hatanakac \\ Masato Naraoka ${ }^{a}$ Akira Munakata ${ }^{a}$ Hiroki Ohkuma ${ }^{a}$ \\ a Department of Neurosurgery, Hirosaki University School of Medicine, Hirosaki, \\ ${ }^{b}$ Department of Neurosurgery, Kuroishi Hospital, Kuroishi, and 'Towada City \\ General Hospital, Towada, Japan
}

\section{Key Words}

Carotid artery stenting · Dilation · Internal carotid artery · Hyperperfusion · Single photon emission computed tomography

\begin{abstract}
Objective: Ischemic stroke and hyperperfusion (HP) are the most frequent and important complications in carotid artery stenosis surgery. Carotid artery stenting (CAS) has replaced carotid endarterectomy in high medical risk patients. Prior to CAS, initial angiographic findings disclose a small caliber internal carotid artery (IC) due to stenosis, but after the stenosis is relieved, the diameter of the IC becomes enlarged. We investigated whether a change in the IC diameter was related to ischemic complication and HP using cerebral blood flow single photon emission computed tomography (SPECT). Methods: From February 2008 to December 2009 we consecutively performed 39 CAS on 35 patients. We retrospectively analyzed the relationship between changes at the level before the entry to the petrous bone canal of the IC and stenosis of the etiological artery, improvement in stenosis, HP and postintervention diffusion-weighted image high-intensity lesions. Statistical analyses comprised Wilcoxon/Kruskal-Wallis analysis, analysis of variance and a multivariate logistic analysis. Results: A total of 9 cases showed HP in SPECT. Severity of IC stenosis and change in the IC at the level before the entry to the petrous bone canal were related with statistical significance to HP. Other factors did not correlate with HP. Conclusion: Procedure-related dilation of the IC at the level before the entry to the petrous bone canal occurred due to release of the etiological stenosis. This finding can also support the prediction of HP.




\section{Introduction}

Carotid artery stenting (CAS) with a filter protection device and a self-expandable open cell stent was approved by the Japanese Ministry of Health, Labour and Welfare following the SAPPHIRE study in 2004 (protected CAS vs. endarterectomy in high-risk patients) [1]. A recent systematic review reported that CAS is significantly better than carotid endarterectomy at avoiding cranial neuropathy and myocardial infarction [2]. Additionally, there is no significant difference in 30-day disability, stroke or death [2]. Ischemic difficulties increased after the use of filter protection devices compared to a balloon protection device due to the fact that a filter protection device does not trap all debris or the oily component of atheroma [3-6]. Ischemic events are the most frequent complications in CAS. Another serious complication is hyperperfusion (HP). HP syndrome is one of the fatal complications of treating carotid artery stenosis. Predictive risk factors for HP have been reported as preoperative ischemic stroke episode, decreased vasoreactivity of the cerebral artery, severe stenosis of the lesion and contralateral internal carotid artery (IC) stenosis [7-11]. The caliber of the IC distal to the stenosis is small due to diminished flow, with the diameter of the IC dilating following the resolution of the IC stenosis.

Our hypothesis was that embolus eluded capture due to the enlargement of the IC diameter, and cerebral blood flow (CBF) excessively improved according to IC enlargement. We investigated whether enlargement of the diameter of the IC at the entrance to the carotid canal is predictive of the development of a postintervention ischemic lesion and/or HP in the perisurgical period. These points have not been discussed in previous reports.

\section{Materials and Methods}

From February 1, 2008 to the end of December 2009 we consecutively performed 39 CAS on 35 patients. The mean age was 69.7 years (IQR 67-72). A total of 28 men and 7 women were included; 4 male patients were operated on bilaterally. We acquired written, informed consent from patients and/or families for interventional procedures and for this study. Preoperative quantified ${ }^{123}$ I-IMP single photon emission computed tomography (SPECT; rest and acetazolamide challenge) according to the autoradiographic method were done within 1 month before the CAS (fig. 1a, left).

Stenosis of the IC was measured with preoperative digital subtraction angiography according to the North American Symptomatic Carotid Endarterectomy Trial criteria. Diameters of the IC at the level before the entry to the petrous bone canal were measured under higher magnification using electric calipers and reference to a guiding catheter by two blinded coauthors who were certificated neurosurgeons and a consensus discussion was held. The IC at the level before the entry to the petrous bone canal is a landmark and this portion is readily monitored during CAS, so we selected this as a reference for any change in the IC diameter.

All CAS procedures were performed by a single, certified operator according to the standard technique, as follows: oral dual or triple antiplatelets were administered (aspirin $100 \mathrm{mg} /$ day, clopidogrel $75 \mathrm{mg} /$ day or cilostazol $200 \mathrm{mg} /$ day). We kept the activated clotting time to more than $300 \mathrm{~s}$ when possible. During CAS, we maintained the patient's systolic blood pressure between 100 and $160 \mathrm{~mm} \mathrm{Hg}$ before stenting, and between 100 and 140 mm Hg after stenting. Filter protection with Angioguard XP ${ }^{\circledR}$ (AGXP; Cordis, N.J., USA) was routinely deployed at the straight-shaped cervicocranial junction of the IC (fig. 1b, arrow head). Prestenting angioplasty was done with a 3-mm-diameter Sterling ${ }^{\circledR}$ balloon (Boston Scientific, Calif., USA). Precise ${ }^{\circledR}$ (Cordis) stents that covered the entire stenotic lesion of etiological relevance were deployed in all cases. Poststenting angioplasties were done with an Aviator ${ }^{\circledR}$ or Amiia ${ }^{\circledR}$ balloon (Cordis) having a diameter estimated to be that of the normal IC. Suction of debris was carried out when no flow occurred. Angiography was done to verify the dilation of the IC (fig. 1c). The position of the image intensifier was maintained during the procedure.

Postoperative management of patients was as follows: argatroban hydrate infused $48 \mathrm{~h}$ after stenting to avoid acute thrombosis. Systolic blood pressure was maintained under $140 \mathrm{~mm} \mathrm{Hg} \mathrm{[12-14].} \mathrm{Diffusion-}$ weighted imaging (DWI) and resting ${ }^{123}$ I-IMP SPECT according to the autoradiographic method were 
a

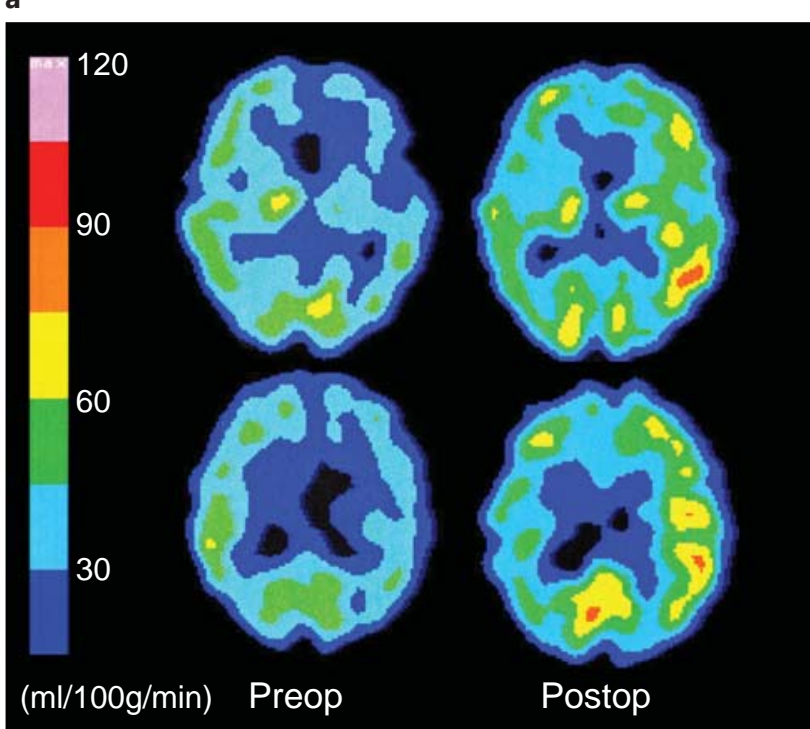

b

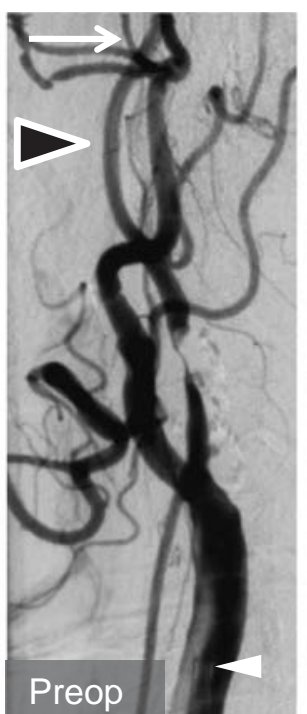

(C) 2013 S. Karger AG, Basel www.karger.com/ine

Shimamura et al.: Dilation of the Internal Carotid Artery at the Entrance to the Carotid Canal following Carotid Artery Stenting Predicts Postprocedural Hyperperfusion

C

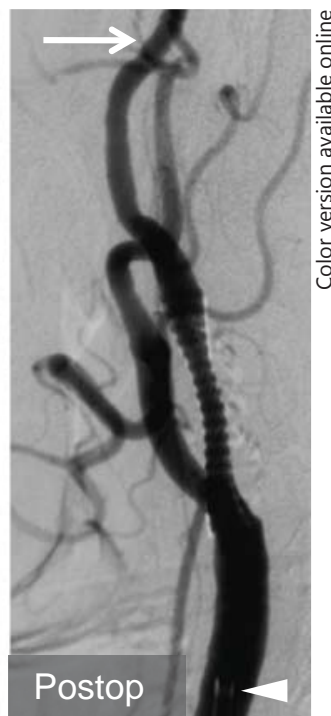

Fig. 1. ${ }^{123}$ I-IMP resting SPECT and angiography. a Preintervention ${ }^{123}$ I-IMP resting SPECT (left) and postintervention ${ }^{123}$ I-IMP resting SPECT (right). Operated left side shows HP. b Preintervention angiography. White arrowhead points to the 8-Fr guiding catheter. White arrow indicates measured IC. Black arrowhead indicates filter landing zone. c Postintervention angiography. White arrowhead points to the 8-Fr guiding catheter. White arrow indicates measured IC.

routinely carried out on the day after CAS (fig. 1a, right). HP was defined as CBF that was increased by at least $100 \%$ compared to preoperative values [14]. When patients showed neurological signs such as seizure, deterioration of consciousness level or focal neurological deficit without cerebral infarction, we defined this as the HP syndrome $[9,3,15]$.

Measurement of the diameter of the IC at the level before the entry to the petrous bone canal and neurological examinations were done by two blinded coauthors who were certificated neurosurgeons, and a consensus discussion was held (fig. 1b, c, white arrows). When vasospasm occurred at the landing zone of the filter device, we waited 3 min or longer for the artery to relax. We did not use a vasodilator for release of vasospasm. Time points of measurement of the IC diameter were the preinsertion of the filter device and after the filter device was pulled out without vasospasm.

We analyzed the relationship between the incidence of procedure-related post-CAS DWI high-intensity lesion or HP and patient characteristics (age, gender, degree of stenosis, hypertension, diabetes mellitus, preoperative DWI high lesion, poor vascular reservation, degree of improvement in stenosis and dilation of the IC at the level before the entry to the petrous bone canal, incidence of no or slow flow, change in the stenosis of the etiological zone, neurological deficits and incidence of HP).

We did a Wilcoxon/Kruskal-Wallis nominal variable analysis, a continuous variable analysis of variance and a multivariate logistic analysis (JMP 8.0.1; SAS Institute, Cary, N.C., USA). A p value $<0.05$ was accepted as statistically significant.

\section{Results}

Preintervention IC stenosis and the diameter of IC at the level before the entry to the petrous bone canal were $74 \% \pm 16 \mathrm{SD}$ and $3.6 \mathrm{~mm} \pm 0.8 \mathrm{SD}$. Postintervention stenosis of the IC and diameter of the IC at the level before the entry to the petrous bone canal were $11 \% \pm$ $14 \mathrm{SD}$ and $4.0 \mathrm{~mm} \pm 0.7 \mathrm{SD}$, respectively. Mean dilation of the IC at the level before the entry to the petrous bone canal was $0.45 \mathrm{~mm} \pm 0.54 \mathrm{SD}$. 


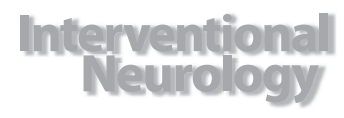

Shimamura et al.: Dilation of the Internal Carotid Artery at the Entrance to the Carotid Canal following Carotid Artery Stenting Predicts Postprocedural Hyperperfusion
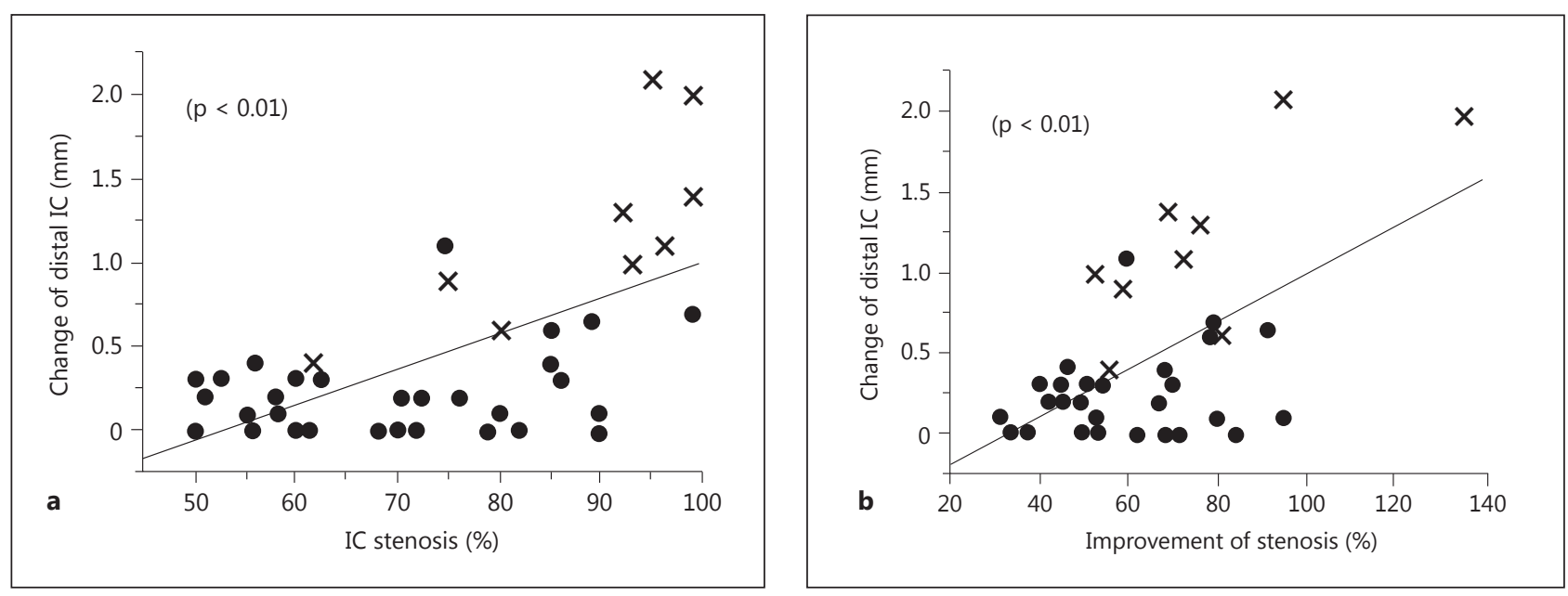

Fig. 2. Relationship between change in diameter of the IC at the level before the entry to the petrous bone canal and the 3 respective parameters. a Preintervention IC stenosis according to NASCET criteria. b Improvement in carotid artery stenosis, calculated as preoperative stenosis minus postoperative stenosis. c Occurrences of HP according to ${ }^{123}$ I-IMP SPECT. These 3 parameters are significantly correlated with dilation of the IC at the level before the entry to the petrous bone canal. Change of distal IC: IC at the level before the entry to the petrous bone canal. - Case of non-HP; $\mathrm{x}=$ case of HP.

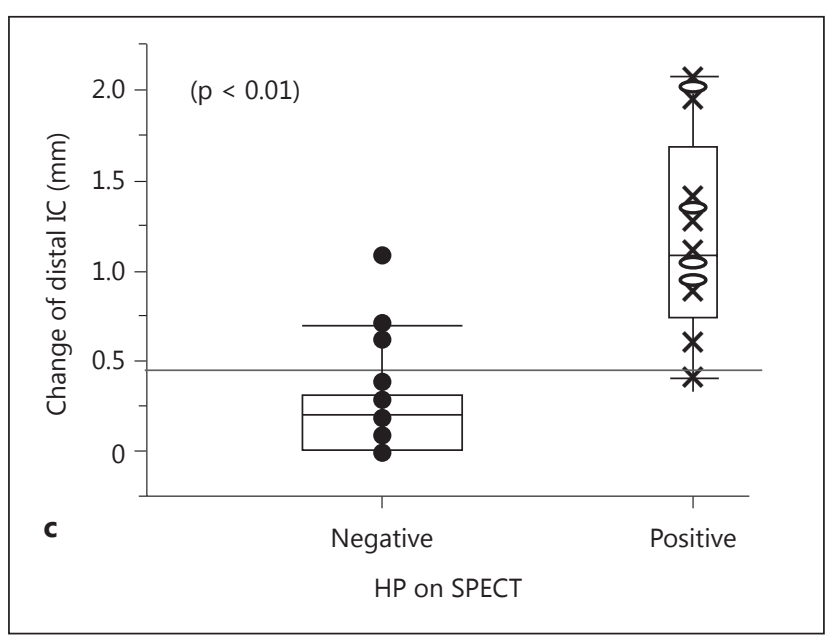

Post-PTA suction of debris was done in 4 procedures due to the slow-flow or no-flow phenomenon (10\%), including 1 transient neurological deficit (2.6\%). No patient suffered a permanent deficit. The incidence of postintervention DWI high-intensity spot lesions, however, was 35\%. A postoperative, new DWI high-intensity lesion did not relate to dilation of the IC.

Overall, 9 cases of 39 procedures experienced the HP phenomenon, whereby IMP-SPECT on the day after CAS revealed the HP. Symptomatic HP appeared in 1 case $(2.6 \%)$ with disturbance of consciousness and restlessness $10 \mathrm{~h}$ after the CAS. The patient's Glasgow coma scale decreased from 15 to 14.

Postoperative subarachnoid hemorrhage and intracerebral hemorrhage did not occur.

Preintervention etiological IC stenosis and a change in IC at the level before the entry to the petrous bone canal significantly correlated with HP (fig. 2). According to logistic analysis of the change in the IC at the entry to the carotid canal and HP, a $0.81-\mathrm{mm}$ enlargement of the IC is associated with a $50 \%$ risk of HP.

The significant differences in the characteristics of patients with or without HP were the preoperative degree of IC stenosis and dilation of IC at the level before the entry to the petrous bone canal (table 1; fig. 2), but only the latter significantly correlated with the occurrence of HP using a multivariate logistic analysis. 
Table 1. Characteristics of patients with and without HP

\begin{tabular}{lcc}
\hline Characteristics & with HP & without HP \\
\hline Age, years & $69.2 \pm 3.0$ & $70.2 \pm 8.4$ \\
Gender (M/F) & $7 / 2$ & $25 / 5$ \\
Preoperative DWI high lesion (yes/no) & $3 / 5$ & $12 / 15$ \\
Decreased resting CBF (80\% of normal) & & $12 / 18$ \\
$\quad$ and vascular reservation $\leq 10 \%$ (yes/no) & $5 / 4$ & $7 / 23$ \\
Diabetes mellitus (yes/no) & $3 / 6$ & $22 / 8$ \\
HP (yes/no) & $7 / 2$ & $5 / 25$ \\
Occlusion of contralateral IC (yes/no) & $0 / 9$ & $70.0 \pm 14.1$ \\
Preoperative degree of IC stenosis, \%* & $88.0 \pm 12.9$ & $10.9 \pm 10.2$ \\
Postoperative degree of IC stenosis, \% & $10.8 \pm 22.3$ & $0.2 \pm 0.3$ \\
Dilation of IC at the level before the entry to the petrous bone canal, mm* & $1.2 \pm 0.6$ & \\
\hline
\end{tabular}

Values are expressed as mean \pm SD unless otherwise indicated. ${ }^{*} \mathrm{p}<0.005$

\section{Discussion}

Our new finding is useful for the prediction of HP. According to our results, we can intensively treat patients who are susceptible to the HP syndrome. We are able to prevent HP syndrome immediately after CAS and do not need additional, expensive instruments or complicated maneuvers. A less expensive prediction of HP syndrome can be done by noting a change in the IC at the level before the entry to the petrous bone canal. When vasodilator is applied to the patient during the procedure, measurement of the IC diameter is not reliable due to drug-induced vasodilation. A gentle surgical maneuver is important to avoid iatrogenic vasospasm.

In all cases, ultrasound scans of the etiological lesion were done on the day after the procedures. All operated sides were followed up during 6-12 months after the CAS with digital subtraction angiography or CT angiography. No cases showed additional dilation of the IC at the entry to the carotid canal after CAS. Dynamic change of the IC occurred during CAS $[16,17]$ and thus a change in IC at the entry to the carotid canal can predict HP.

According to logistic analysis of the change in the IC at the entry to the carotid canal and HP, a $0.81-\mathrm{mm}$ enlargement of the IC is associated with a 50\% risk of HP. We must take into consideration that a change in the IC as small as $<0.4 \mathrm{~mm}$ (about $10 \%$ of the normal diameter of the IC) includes a measurement error, but a case involving this small a change did not lead to HP. In the case of optimal collateral from the circle of Willis and/or leptomeningeal anastomosis, the change in the IC diameter was small after relieving severe IC stenosis [16]. We speculate that decreased vasoreactivity of the cerebral artery reflects a change in the IC at the entry to the carotid canal.

Ogasawara et al. [15] reported that HP can be detected immediately after carotid endarterectomy by SPECT, and Matsumoto et al. [18] reported that the increase in CBF detected just after CAS using near-infrared spectroscopy predicts the cerebral HP syndrome. HP starts during carotid artery stenosis surgery, and we detected dynamic IC dilation due to an increase in blood flow that can induce the HP syndrome. Transcranial Doppler sonography is less invasive and can be repeated without side effects but it does not always detect CBF, so physicians cannot screen all patients.

Dilation of the IC at the level before the entry to the petrous bone canal did not correlate with the appearance of DWI high-intensity lesion. In our series, the $95 \% \mathrm{CI}$ of the filter landing 
Shimamura et al.: Dilation of the Internal Carotid Artery at the Entrance to the Carotid

zone diameter was 3.4-3.9 mm, so an AGXP with a diameter of $5 \mathrm{~mm}$ was able to capture debris during CAS surgery. AGXP can catch debris after dilation of the IC within their capacity to expand. The incidence of postoperative DWI high-intensity signals is similar to previously reported results [19-24]. Our cases did not suffer major ischemic deficit, but we need to make an effort to reduce the DWI high-intensity lesion. In this study we did not use other embolic protection devices, so our results from DWI are not directly beneficial for all CAS cases. Additionally, we need to consider that incidence of DWI high signal after the CAS is correlated with plaque vulnerability [25-27].

We routinely used argatroban sulfate after CAS. According to previous reports, heparin infusion should be done during intervention, but the definitive medication after stenting was not reported [1]. Argatroban sulfate is safer than heparin because this drug is effective for patients without antithrombin III, and heparin induces thrombocytopenia. Also, the half-life of argatroban sulfate is shorter than that of heparin.

The patient number in our study is small. A large study is necessary to confirm our findings.

\section{Conclusion}

From our investigation, the dilation of the IC at the level before the entry to the petrous bone canal predicts HP on IMP-SPECT, while incidence of postoperative DWI high-intensity lesion does not relate to IC dilation.

\section{Acknowledgments}

We thank Mark Inglin (University of Basel) for his editorial assistance. This work was supported by JSPS KAKENHI grant number 40312491 to N.S.

\section{References}

1 Yadav JS, Wholey MH, Kuntz RE, et al: Protected carotid-artery stenting versus endarterectomy in high-risk patients. N Engl J Med 2004;351:1493-1501.

- 2 Ederle J, Featherstone RL, Brown MM: Randomized controlled trials comparing endarterectomy and endovascular treatment for carotid artery stenosis: a Cochrane systematic review. Stroke 2009;40:1373-1380.

3 Skjelland M, Krohg-Sorensen K, Tennoe B, Bakke SJ, Brucher R, Russell D: Cerebral microemboli and brain injury during carotid artery endarterectomy and stenting. Stroke 2009;40:230-234.

- 4 Schluter M, Tubler T, Mathey DG, Schofer J: Feasibility and efficacy of balloon-based neuroprotection during carotid artery stenting in a single-center setting. J Am Coll Cardiol 2002;40:890-895.

- 5 Tubler T, Schluter M, Dirsch 0, et al: Balloon-protected carotid artery stenting: relationship of periprocedural neurological complications with the size of particulate debris. Circulation 2001;104:2791-2796.

- 6 Bonati LH, Jongen LM, Haller S, et al: New ischaemic brain lesions on MRI after stenting or endarterectomy for symptomatic carotid stenosis: a substudy of the International Carotid Stenting Study (ICSS). Lancet Neurol 2010;9:353-362.

7 Grunwald IQ, Politi M, Reith W, et al: Hyperperfusion syndrome after carotid stent angioplasty. Neuroradiology 2009;51:169-174.

- 8 Hosoda K, Kawaguchi T, Ishii K, et al: Prediction of hyperperfusion after carotid endarterectomy by brain SPECT analysis with semiquantitative statistical mapping method. Stroke 2003;34:1187-1193.

9 Kaku Y, Yoshimura S, Kokuzawa J: Factors predictive of cerebral hyperperfusion after carotid angioplasty and stent placement. AJNR Am J Neuroradiol 2004;25:1403-1408.

10 Morrish W, Grahovac S, Douen A, et al: Intracranial hemorrhage after stenting and angioplasty of extracranial carotid stenosis. AJNR Am J Neuroradiol 2000;21:1911-1916.

11 Ogasawara K, Sakai N, Kuroiwa T, et al: Intracranial hemorrhage associated with cerebral hyperperfusion syndrome following carotid endarterectomy and carotid artery stenting: retrospective review of 4,494 patients. J Neurosurg 2007;107:1130-1136. 
Shimamura et al.: Dilation of the Internal Carotid Artery at the Entrance to the Carotid

Canal following Carotid Artery Stenting Predicts Postprocedural Hyperperfusion

12 Abou-Chebl A, Yadav JS, Reginelli JP, Bajzer C, Bhatt D, Krieger DW: Intracranial hemorrhage and hyperperfusion syndrome following carotid artery stenting: risk factors, prevention, and treatment. J Am Coll Cardiol 2004;43:1596-1601.

13 Abou-Chebl A, Reginelli J, Bajzer CT, Yadav JS: Intensive treatment of hypertension decreases the risk of hyperperfusion and intracerebral hemorrhage following carotid artery stenting. Catheter Cardiovasc Interv 2007; 69:690-696.

14 Piepgras DG, Morgan MK, Sundt TM Jr, Yanagihara T, Mussman LM: Intracerebral hemorrhage after carotid endarterectomy. J Neurosurg 1988;68:532-536.

$\checkmark 15$ Ogasawara K, Yukawa H, Kobayashi M, et al: Prediction and monitoring of cerebral hyperperfusion after carotid endarterectomy by using single-photon emission computerized tomography scanning. J Neurosurg 2003;99:504-510.

16 Chuang YM, Lin CP, Wong HF, et al: Plasticity of circle of Willis: a longitudinal observation of flow patterns in the circle of Willis one week after stenting for severe internal carotid artery stenosis. Cerebrovasc Dis 2009; 27:572-578.

17 Fan X, Zhu H, Chen X, et al: The impact of carotid angioplasty and stenting on the cerebrovascular reactivity. Cerebrovasc Dis 2012;34:13-17.

18 Matsumoto S, Nakahara I, Higashi T, et al: Near-infrared spectroscopy in carotid artery stenting predicts cerebral hyperperfusion syndrome. Neurology 2009;72:1512-1518.

19 Asakura F, Kawaguchi K, Sakaida H, et al: Diffusion-weighted MR imaging in carotid angioplasty and stenting with protection by the reversed carotid arterial flow. AJNR Am J Neuroradiol 2006;27:753-758.

20 Cosottini M, Michelassi MC, Puglioli M, et al: Silent cerebral ischemia detected with diffusion-weighted imaging in patients treated with protected and unprotected carotid artery stenting. Stroke 2005;36:2389-2393.

-21 Roh HG, Byun HS, Ryoo JW, et al: Prospective analysis of cerebral infarction after carotid endarterectomy and carotid artery stent placement by using diffusion-weighted imaging. AJNR Am J Neuroradiol 2005;26:376384.

22 Schluter M, Tubler T, Steffens JC, Mathey DG, Schofer J: Focal ischemia of the brain after neuroprotected carotid artery stenting. J Am Coll Cardiol 2003;42:1007-1013.

$\checkmark 23$ Ghorab K, Macian F, Adoukounou T, Magy L, Chapot R, Vallat JM: Carotid angioplasty stenting revisited: clinical and radiological (MRI) outcome. Cerebrovasc Dis 2008;25:21-25.

24 Rosenkranz M, Thomalla G, Havemeister S, et al: Older age and greater carotid intima-media thickness predict ischemic events associated with carotid-artery stenting. Cerebrovasc Dis 2010;30:567-572.

-25 Rosenkranz M, Wittkugel 0, Waiblinger C, et al: Cerebral embolism during carotid artery stenting: role of carotid plaque echolucency. Cerebrovasc Dis 2009;27:443-449.

-26 Rosenkranz M, Russjan A, Goebell E, et al: Carotid plaque surface irregularity predicts cerebral embolism during carotid artery stenting. Cerebrovasc Dis 2011;32:163-169.

27 Yamada K, Yoshimura S, Kawasaki M, et al: Prediction of silent ischemic lesions after carotid artery stenting using virtual histology intravascular ultrasound. Cerebrovasc Dis 2011;32:106-113. 